\title{
Die weltweite Verbreitung des Monograptus priodon Bronn als Beitrag zur Paläogeographie des Graptolithenmeeres.
}

\author{
Von Rudolf Hundt.
}

Es liegt in der pseudoplanktischen Lebensweise mancher Graptolithenarten mit begründet, daß sie über große Flächen der Erde verbreitet sind. Diese weltweite Verbreitung trifft für die gesamte Graptolithenfamilie $\mathrm{zu}$, während natürlich einzelne Arten mehr oder weniger umgrenzte Lebensbezirke besaßen.

Von den obersilurischen Graptolithen hat nach meinen Untersuchungen Monograptus priodon BRoNs, der zuerst und am häufigsten in der LAPwOBTeschen Zone 15 (nach ELLES und Wood 22-23), dem Tarannon, auftritt, die größte flächenhafte Verbreitung gehabt. Bei ihm ist die Thekenbildung so charakteristisch, daß er am leichtesten auch von den Autoren erkannt werden mußte, die sich nicht eingehend nur mit Graptolithen befaßt haben.

Man hat von Monograptus priodon Brown eine Menge Mutationen und Varianten abgezweigt, die sich zum großen Teil als Verdrückungs- oder Einbettungsformen erwiesen haben. Oft ist in der Faunenliste eines Fundortes, einer Gegend nur diese eine Art genannt worden, so daß in diesem oft einzigen und alleinigen Vorkommen die Häufigkeit dieser Art in Zone 15 an deutschen, englischen, böhmischen und schwedischen Fundstellen ihre Bestätigung auch hinsichtlich der Weltverbreitung findet. Zugegeben, daß in den verarbeiteten Faunenlisten hier und da in der Bestimmung Fehler vorgekommen sind, so mag dies aber bei $M . p$. BRoNN weniger als bei allen anderen Arten gesehehen sein, denn seine Artmerkmale sind zu charakteristisch. Zeigt sich schon in den schiefrigen Fazies der Tarannonzeit $M$. p., so gewinnt das Silurmeer zu dieser Zeit noch gewaltig an Raum, wenn man die kalkigen Äquivalente gleichen Alters in den außerdeutschen Ländern in diese Betrachtung aufnehmen würde, in denen eine Nichtgraptolithenfauna eingeschlossen bewahrt wird.

Man kann sagen, daß fast an allen Stellen der Erde, an denen graptolithenführendes Obersilur aufgefunden werden konnte, $M . p$. nachgewiesen worden ist.

Wenn eine Art eine solche weltweite Verbreitung besitzt, so ist es doch wohl am wahrscheinlichsten, daß man für sie eine pseudoplanktische Lebensweise annimmt oder ihr in Grobkolonien, wie es Funde von Frech an sejnem Linograptus Nilssoni von Herzogswalde, KIRSTE an Nilssoni-artigen einreihigen Formen vom Johannisberg bei Ronneburg und Verfasser an Monograptus vomerinus Nich. und vielleicht auch an Monograptus riccartonensis Lapw. am Willenberg bei Schönau im Bober-Katzbachgebirge gezeigt haben, eine größere Bewegungsfreiheit einräumt. Jedenfalls trifft die Annahme Mancks, die Monograpten seien an harten Körpern festgewachsen gewesen oder die WMaNs, sie hätten vin der tieferen Litoralregion zusammenhängende, wehende Rasen « gebildet, bei dem weltweit verbreiteten $M$. p. Brons nicht zu.

Man kann auch nicht gut annehmen, daß nur die Sikulae eine weltweite Verbreitung der Art ermöglichten und die ausgewachsenen Kolonien später als sessiles Benthos lebten.

Nun findet sich in der Zone 14 ein Vorläufer des $M . p$. in dem $M$. Becki Barr., der ihm im Zellenbau und auch in Gesamthabitus oft zum Verwechseln nahekommt. Aus der Literatur ist mir allerdings eine weltweite Verbreitung dieser Art nicht bekannt geworden, wenn man sie auch häufig an vielen Fundpunkten der Erde findet. Immerhin wäre es möglich, daß an verschiedenen Stellen unter gleichen Lebensbedingungen - große klimatische' Unterschiede fehlten dem Silurmeer wohl völlig - aus $M$. Becki Barr. sich $M . p$. BronN entwickelte und von diesen Entstehungszentren nach den entlegenen Meeresteilen wanderte. 
Wenn man nun an manchen Stellen in den Faunenlisten Vertreter von Rastriten und Retioliten genannt findet, so ist es nicht ausgeschlossen, daß dort auch $M . p$. BronN vorhanden gewesen ist. Wenn auch meines Wissens in den jüngsten Ruastritesschichten, in der Lapwormischen Zono 14 kein $M . p$. BroNN vorkommt, so bleibt aber doch die Möglichkeit bestehen, daß in den Übergängen zu Zone 15 nicht erkannte, vielleicht noch zu $M$. Becki gerechnete $M$. p. sich zeigen.

Nachstehend seien die weltweit verbreiteten Fundorte von M. p. Brown genannt. Deutschland: Westthüringisches Schiefergebirge zwischen Hämmern, Großbreitenbach, Saalfeld, Ludwigstadt. Ostthüringisches Schiefergebirge zwischen Gera, Ronneburg, Weida, Loitzsch-Hohenleuben. Frankenwald und Fichtelgebirge zwischen Lehesten, Goldkronach, Naila, Hof, Blankenstein a. d. Saale, Schleiz. Nach den GüMBELschen Abbildungen in seiner Geognostischen Beschreibung des Fichtelgebirges, S. 447, Fig. 4-5 sind viele seiner Angaben, nach denen $M$. colonus BARR. vorkommen soll, irrtümlich, denn er bildet an dieser Stelle $M . p$. BRONN ab. Auf S. 412 gibt die Abbildung von $M$. colonus BARR, ein richtiges Bild von dieser Art. Sachsen. Im sächsischen Vogtland zwischen Triebes bis nach Plauen und im übrigen. Könıreich Sachsen, im Elbtalschiefergebiet zwischen Kreischa, BerggieBhübel und Gottleuba. Lausitz und Schlesien von Lauban bis zum östlichen Vorkommen bei Herzogswalde. Harz. Im Selkegebiet und Nachbargebieten ist Tarannon mit $M$. p. nachgewiesen. Kellerwald. Die Untersuchungen von WEDEKrMD liegen noch nicht vor. Aber da Tarannon nachgewiesen ist, wird sich sowohl in den Steinhorner als auch in den Urfer Schichten $M . p$. zeigen. Gießen (Lindener Mark). Die Ähnlichkeit der Schichten der Lindener Mark mit denen des Kellerwaldes lassen auch von hier $M$. p.-Funde erwarten.

Polen: Polnisches Mittelgebirge bei Zbza and Sandomierz.

Böh men: Im ganzen Silurbecken.

Alpen: Osternigabschnitt in Kärnten und in den Karnischen Alpen.

Bulgarien: Das im Distrikt Sofia als massenhaft bemerkte Vorkommen von Monograpten, die von Aluachverdjefr in die Gruppe des $M$. colonus BarR. eingereiht werden, kann möglicherweise $M . p$. enthalten.

Belgien: Im Massiv von Brabant im Sambre-Maasgebiet ist $M . p$. nachgewiesen.

Frankreich: In folgenden französischen Landschaften fand sich $M . p_{.}$: Languedoc, Pyrenäen, Normandie, Bretagne.

England: Verbreitung besitzt $M . p$. in Nord- und Zentral-Wales, im Seedistrikt und in Südschottland.

Däne mark: An der Südküste Bornholms kommen Rastriten- und Cyrtograptusschiefer vor.

Schweden: $M . p$. ist in den schwedischen Graptolithenschieferfaunen vorhanden (Westergotland, Gotland usw.).

Spanien: An verschiedenen Stellen (Catalonien, Sierra Morena, Arragonien, Pyrenäen) ist $M . p$. gefunden whrden.

Portugal: Die portugiesischen Silurschichten lieferten DeLGado an vielen Fundorten $M . p$.

Sardinien: DE la Marmora exwähnt in seiner Voyage en Sardaigne den $M . p$.

Afrika: Marokkos hoher Atlas hat am Ait Mdirual und bei Haci-el-Khenig $M, p$. geliefert.

Südamerika: Im Maecura-Gestein des Amazonasgebiet wurden nach Kurzen Mon. clintonensis-ähnliche Graptolithen gefunden. Ich werde unter Nordamerika auf meine Ansicht über Mon. clintonensis zurückkommen.

Nordamerika: In den oberen Clinton-Schichten New Yorks kommt nach RUEdemann $M$. clintonensis CARR. vor. Nach den Abbildungen zu urteilen und nach dem Zusammenvorkommen mit Retiolites Geinitzianus BARR. var. venosus HaLl (venosus scheint mir keine besondere Variante von Ret. Geinitzianus za sein), ist $M$. clintonensis als $M . p$. aufgefaBt. Er kommt nur im 
Staate New - York vor und ist wim Golf von Mexiko oder im Süd-Atlantik ausgebildet, der zu jener Zeit unzweifelhaft mehr oder weniger vom Nord-Atlantik getrennt war \&. RUEDEMATN führt aus dem Verbreitungsgebiet des M. clintonensis noch eine andere Art: $M$. p. mat. chapmanensis RUED. an, den ich für eine Variante von $M . p$. halte.

Grönland: Es waren schon früher Monograpten (convolutus) bekannt geworden, aber es besteht die Vermutung, daß unter dem Material der II. Thule-Expedition 1916-1918 nach Nord-Grönland, die an den Küsten des KennedyKanals, im Washington- und Hall-Land Obersilur (Pentamerus-Kalk, Rastriten und Monograpten) entdeckte, sich $M$. $p$.-Reste zeigen werden.

China: RroнTHоFE⿰冫 berichtet über obersilurische Graptolithenschiefer am Nordabhang des Lunschan bei Tschinkiang. Die Faunenliste läßt schließen, daß sich unter den vertretenen Zonen auch die Schichten, in denen $M . p$. zu Hause ist, nicht fehlen.

Australien: Die aus dem südlichen Teil von Neu-Süd-Wales und an der Grenze von Viktoria angeführten Diplograptus palmeus und Monograptus dubius kommen an anderen, genau durchforschten Gebieten mit $M$. p. zusammen vor, so daß bei genauerem Suchen sich auch hier $M . p$. zeigen wird.

Wenn auch nicht an allen Stellen $M . p$. selbst in den Faunenlisten aufgeführt wird, so berechtigt aber doch die Begleitfauna, die man von ihm aus gut durchforschten Gebieten genau kennt, anzunehmen, da $B$ er entweder übersehen worden ist oder aber noch nicht aufgefunden wurde, denn einmal sind die Graptolithenschichten nicht sehr mächtig, zum andern ist die Versteinerungsart der Graptolithen mitunter nicht sehr dauerhaft. Nach den bisher bekannt gewordenen obersilurischen Graptolithenfundorten kommt nur der in Bolivia liegende überhaupt nicht in Frage, von vornherein vèrdächtig zu sein, den $M . p$. zu liefern.

Zieht man die gleichaltrigen, kalkigen Äquivalente mit ein in diese Betrachtung, dann scheint das Silurmeer in der Tarannonzeit, in der $M$. $p$. seine größte Entwicklung hinsiohtlich der Individuenerzeugung durchmachte, in der er weltverbreitet war, seine größte Flächenausdehnung erreicht zu haben. Das Untersilurmeer kannte noch keine solche umfassende Größe wie das Obersilurmeer. Frecr schreibt in seiner Lethaea geognostica S. 102: Die am Schlusse der Untersilurzeit eröffneten Meeresverbindungen erweitern sich und bedingen eine so allgemeine Verbreitung der Meeresfauna, wie sie aus wenigen Abschnitten der Erdgeschichte bekannt ist. \&

\title{
Zum Thema Kramenzelkalk.
}

\author{
Eine Erwiderung an O. H. Schindewolf.
}

Von A. Born (Frankfurt a. M.).

Im Heft 1-2, Bd. 12, der Geologischen Rundschau veröffentlicht $\mathrm{O} . \mathrm{H}$. Schindewolf zum Thema Kramenzelkalk einen Aufsatz, der im wesentlichen über eine 1912) von mir gegebene Zusammenstellung über dieses Thema nur insofern hinausgeht, als wir erfahren, daß 0 . H. SOHINDEwoLF einerseits mit meiner Anschauung der Kramenzelkalkgenese nicht einverstanden, andererseits sich zu der von ZIRKFi 1894 ausgesprochenen Auffassung bekennt.

1) A. BorN, Die geologischen Verhältnisse des Oberdevons im Aecketal. N. Jahrb. f. Min. B. Bd. 34. 1912. S. 62. - Über die Entstehung von Kramenze]kalk. 5. Jahresber. niedersächs. geologischer Ver. Hannover. 1912. S. 1. 